\title{
Transitionless Enhanced Confinement and the Role of Radial Electric Field Shear
}

\author{
D. R. Ernst, R. E. Bell, M. G. Bell, R. V. Budny, B. Coppi, ${ }^{*}$ W. M. Dorland ${ }^{\dagger}$ G. W. Hammett,
} R. J. Hawryluk, K. W. Hill, M. T. Kotschenreuther, ${ }^{\ddagger}$ D. K. Mansfield, D. R. Mikkelsen, H. K. Park, M. Porkolab, * S. D. Scott, G. Schmidt, E. J. Synakowski, M. C. Zarnstorff, and the TFTR Group Princeton University Plasma Physics Laboratory, MS34, P.O.B 451, Princeton, NJ 08543

* Massachusetts Institute of Technology, Cambridge, Massachusetts

${ }^{\dagger}$ Institute for Plasma Research, University of Maryland, College Park, Maryland

${ }^{\ddagger}$ Institute for Fusion Studies, University of Texas, Austin, Texas

(Submitted November 19, 1998, accepted for publication in Phys. Plasmas October 1999)

\begin{abstract}
Evidence for the role of radial electric field shear in enhanced confinement regimes attained without sharp bifurcations or transitions is presented. Temperature scans at constant density, created in the reheat phase following deuterium pellet injection into supershot plasmas in the Tokamak Fusion Test Reactor [J. D. Strachan, et al., Phys. Rev. Lett. 58, 1004 (1987)] are simulated using a first-principles transport model. The slow reheat of the ion temperature profile, during which the temperature nearly doubles, is not explained by relatively comprehensive models of transport due to Ion Temperature Gradient Driven Turbulence (ITGDT), which depends primarily on the (unchanging) electron density gradient. An extended model, including the suppression of toroidal ITGDT by self-consistent radial electric field shear, does reproduce the reheat phase.
\end{abstract}

PACS numbers: 52.55.Dy, 52.25.Fi, 52.30.-q, 52.65.Tt 


\section{INTRODUCTION}

The complete suppression of turbulence by radial electric field shear is thought to be the cause of spatially and temporally lomathcalized transitions to improved confinement in the H-Mode (high confinement mode), VH-Mode (very high confinement mode), and weak or reversed magnetic shear regimes, as reviewed in Ref. [1]. Results specific to TFTR are reviewed in Refs. [2, 3, 4, 5]. However, regimes exhibiting similar degrees of improvement, such as the TFTR supershot, are attained continuously, without transition phenomena or reversed magnetic shear. The absence of a clear transition or other obvious temporal signature, together with the proximity to marginal stability to toroidal ion temperature gradient driven modes, requires a more careful treatment to determine the relevance of radial electric field shear. Comparisons of radial electric field shearing rate and maximum linear growth rate, usually carried out at the time of transition to enhanced confinement, may not be conclusive near marginal stability where the linear growth rate is very sensitive to small errors in measured temperature and density profiles. Accordingly, a closed-loop, nonlinear simulation, which can more accurately describe the deviation from ITG marginal stability, allowing the temperature profile to relax, is required.

Supershot plasmas show several disparate confinement trends which appear to be consistent with the proposed mechanism of turbulence suppression by intrinsically generated (as well as externally applied) sheared flows, described in Refs. [6, 7, 8]. In this paper, pellet perturbation experiments are considered in detail. During recovery from these perturbations, the effects of the density gradient on supershot confinement and toroidal ITG marginal stability remain unchanged, in the sense that a temperature scan at constant density follows the relatively rapid recovery of the density profile within the same discharge.

A model invoking $E_{r}$ shear suppression of toroidal ion temperature gradient driven turbulence has been recently described $[6,7]$. This model, developed prior to the availability of poloidal rotation data, relies on a self-consistent neoclassical calculation of the poloidal velocity. Simulations of supershots using this model show that the core ion temperature is consistent with a simple criterion for near-complete turbulence suppression by $E_{r}$ shear, which expresses a balance between turbulence growth and suppression.

In original presentation of this paper, ${ }^{1}$ we examined new measurements of the carbon poloidal rotation in supershot plasmas. A significant discrepancy with neoclassical estimates existed, and the apparent poloidal rotation was characterized with a simple empirical scaling proportional to the ion temperature. This scaling was used, in place of our prior neoclassical calculations, to simulate supershot temperature profiles in discharges for which poloidal rotation data was unavailable. However, following the presentation of the results (some of which have been preserved in the Appendix), a new effect was discovered [9], involving the finite lifetime of the observed carbon $5+$ VIII to VII line emission and the energy dependence of the excitation cross-section in magnetized plasmas. The effect is significant when the radiative lifetime of the observed spectral line is comparable to the period of gyration of the emitting atom, i.e., at high ion temperatures in strong magnetic fields. Preliminary investigation shows this effect is large enough to change the sign of the apparent carbon poloidal velocity, changing the interpretation of spectroscopic poloidal rotation measurements at high temperatures. In addition, initial revised analysis of poloidal rotation data, accounting for the new effect, suggests that the carbon poloidal rotation may be quite small in supershot as well as L-Mode plasmas. This is in qualitative agreement with our neoclassical calculations. Therefore, we have reverted to a neoclassical calculation of the carbon poloidal velocity as in our prior model, because no revised poloidal rotation data is presently available. Further, a growing body of evidence suggests that neoclassical parallel momentum exchange processes are relevant in regimes with strongly reduced anomalous transport [10,12].

Following a pellet perturbation during neutral beam heating in a supershot plasma, the density

\footnotetext{
${ }^{1}$ Invited Talk J5I1.04, APS Annual Meeting of the Division of Plasma Physics, New Orleans, November 11-17, 1998
} 
profile recovers relatively quickly and completely, while the ion temperature profile suffers a large and lasting degradation, demonstrating hysteresis of the energy confinement time $\tau_{E}$ with respect to the peaking factor $n_{e}(0) /\left\langle n_{e}\right\rangle$ (little effect is seen on $T_{e} \ll T_{i}$ ), and violating the empirical scaling $\tau_{E} / \tau_{E}^{89 P} \sim n_{e}(0) /\left\langle n_{e}\right\rangle$, where $\tau_{E}^{89 P}$ is the L-Mode scaling. The stored energy eventually recovers, forming an ion temperature scan at constant density. This difference in recovery timescales for the density and stored energy was first observed in Ref. [13] for the case of carbon pellet perturbations. That study confirmed profile resiliency of the electron temperature [14] following the pellet, in addition to observing that the electron stored energy actually increases transiently as a result of the perturbation. Most interesting, however, was that in contrast with the large degradation and slow recovery suffered by supershots, L-Mode plasmas suffered no loss of stored energy during deep carbon pellet perturbations.

\section{DESCRIPTION OF MODEL}

The ion temperature profiles are calculated using a new transport simulation code TRV [6] which necessarily improves numerical convergence to accomodate the strongly increased "stiffness" resulting from $E_{r}$ shear. The code is capable of a variety of calculations, including self-consistent evolution of numerically evaluated mutispecies neoclassical velocities [10], as well as simultaneous evaluation of classical differences in multispecies ion temperatures. The latter is often important in lower density in supershot plasmas; the calculated hydrogenic temperature gradient scale length, relevant to the toroidal ITG mode, often differs from that of the impurity. The effect of $E_{r}$ shear on the ion thermal diffusivity is approximated by taking a simple parameterization of the results nonlinear flux-tube gyrofluid calculations with adiabatic electrons [15]. These calculations show that, in the absence of shear in the parallel velocity, that turbulent transport is completely suppressed according to the criterion that the $E_{r}$ shearing rate $\omega_{E \times B}$ for isotropic turbulence $[16,17]$ exceeds the linear growth rate of the most unstable mode $\gamma_{\text {lin }}^{\max }$. It is generally agreed that no great philosophical significance should be attributed to this criterion, and that it is loosely based on the results of numerical simulations (and may miss some parametric dependences). The nonlinear turbulence calculations also suggest that the reduction in $\chi_{i}$ is very roughly linear in the ratio $\left|\omega_{E \times B}\right| / \gamma_{\text {lin }}^{\max }$. These results are parameterized with the following expression [18],

$$
\chi_{i}=\chi_{i 0}\left(1-\alpha_{E}\left|\omega_{E \times B}\right| / \gamma_{\text {lin }}^{\max }\right) \times H\left(1-\alpha_{E}\left|\omega_{E \times B}\right| / \gamma_{\text {lin }}^{\max }\right)+\chi_{i}^{\text {neo }}
$$

where $\chi_{i 0}$ is evaluated using the parameterization of Ref. [19], $H$ is the Heaviside step function, $\chi_{i}^{\text {neo }}$ is the standard neoclassical diffusivity with impurities [20], and the shearing rate on the outer midplane is given by

$$
\omega_{E \times B}=\frac{R B_{\theta}}{B} \frac{d}{d R}\left(\frac{E_{r}}{R B_{\theta}}\right),
$$

where $R$ is the local major radius, $B_{\theta}$ and $B$ are the poloidal and toroidal magnetic fields, and $E_{r}$ is evaluated from the carbon radial force balance. While the parameterization above for including $E_{r}$ shear was included in the model in Ref. [18], the radial electric field itself was not calculated as part of the model, but was approximated by the product of the measured carbon toroidal velocity and poloidal magnetic field. The model we present includes a self-consistent calculation of the radial electric field utilizing all three terms in the radial force balance. The carbon poloidal rotation is evaluated from a multispecies neoclassical calculation [21, 10]. The maximum linear growth rate is evaluated using an extension of the parameterization of Ref. [19]. The constant $\alpha_{E}=1.0$ is the same for all simulations in this paper.

The simulations take as inputs the edge ion temperature boundary condition $T_{i}(r / a=0.85)$, the measured electron and impurity density profiles, the measured electron temperature (which does not play a significant role in the confinement changes studied), and the thermal ion density and power 
deposition profiles inferred from TRANSP Monte Carlo calculations of neutral beam thermalization [22]. Also, a radial convective transport $c_{i} \Gamma_{i} T_{i}$ is assumed, where $\Gamma_{i}$ is the radial ion flux density, and $c_{i}=3 / 2$. The results are generally insensitive to $c_{i}$ except in certain extreme cases. As shown in Refs. [6, 7], the ion temperature profile rises to satisfy the criterion $\alpha_{E}\left|\omega_{E \times B}\right| \simeq \gamma_{\text {lin }}^{\max }$ in the inner half-radius, where convection is important $[6,7,8]$. This lessens the importance of the assumed convective multiplier $c_{i}$, as well as potential inaccuracies in $\chi_{i 0}$.

When the poloidal rotation is inferred from two-species neoclassical calculations, it is evaluated consistently using the simulated ion temperatures. The neoclassical calculations are numerical, using the full viscosity matrix, calculated by integration over velocity space. Simulation of the toroidal velocity would be a trivial extension under the assumption $\chi_{i} \simeq \chi_{\varphi}[23,24]$, where $\chi_{i}$ and $\chi_{\varphi}$ are the diffusivities for ion heat and toroidal angular momentum, respectively. The code is capable of simultaneously calculating neoclassical differences between impurity and hydrogenic toroidal velocities, which reproduces the measured "notch" feature in the carbon toroidal velocity profile [10].

\section{PELLET PERTURBATIONS AND $E_{r}$ SHEAR SUPPRESSION}

\section{III.A GENERAL}

In view of the significant influence of toroidal ITG marginal stability on the ion temperature profile, suggested in Ref. [19], and our finding that $E_{r}$ shear suppression tends to amplify these parametric dependences (while adding some new ones) [7], the question arises as to how the influence of $E_{r}$ shear can be identified experimentally when there is no obvious confinement transition. Frozen deuterium pellets provide a means of perturbing the plasma temperature and density profiles in such a way as to result in a temperature scan within a single discharge, without introducing impurities. The extended reheat observed, following cooling by the pellet, is not explained qualitatively or quantitatively by the linear stability properties of toroidal ITG modes or their known transport characteristics discussed in Ref. [19]. Nor is the reheat with constant density profile consistent with empirical scalings relating the energy confinement time to the peakedness of the electron density or beam heating profile [25, 26, 27, 28].

The critical temperature gradient for destabilizing the toroidal ITG mode depends strongly on the electron density gradient, and relatively weakly on the ratio of ion to electron temperatures, $T_{i} / T_{e}$. Therefore, simulations using the model of Ref. [19], without $E_{r}$ shear, result in ion temperatures that do not evolve significantly following recovery of the electron density profile. This shows that dependences on $T_{i} / T_{e}$ and on the edge ion temperature are not sufficient to explain the reheat beyond the time of the density profile recovery. In addition, other known empirical scalings for supershots, such as the relation $\tau_{E} \propto(H \alpha)^{-0.24}[29]$ are not sufficient to explain the continued reheat given the observed evolution of plasma edge parameters. Therefore, an additional stabilizing mechanism is needed to explain the slow rise of the ion temperature to twice its value following full recovery of the density profile. This significant increase in ion temperature is reproduced by our simulations only when $E_{r}$ shear suppression is included.

Immediately following injection of a deuterium pellet into a supershot plasma, the density profile is flattened, and the temperature decreases to L-Mode levels. Unlike L-Mode plasmas, pellets injected into supershot plasmas result in significant and long duration loss of stored energy. The density profile recovers relatively rapidly, however, followed by a much slower recovery of $T_{i}$ and the global energy confinement time $\tau_{E}$. The latter part of this slow recovery constitutes a temperature scan at constant density. Given the rough neoclassical expression for hydrogenic ions, $E_{r} \simeq-T_{i} / e r_{n}+$ $V_{\varphi i} B_{\theta} \propto T_{i}$ where $r_{n}^{-1}=-d \ln n_{i} / d r$ is the thermal ion density gradient scale length, $r$ is the minor radius, $T_{i}$ is the ion temperature, $V_{\varphi i}$ is the ion toroidal velocity, $B_{\theta}$ is the poloidal magnetic field, and the units are MKS-keV. With $V_{\varphi i} \propto T_{i}$ as typically observed at high rotation velocities, a situation results during reheat in which $T_{i}$ and $E_{r}$ evolve simultaneously and independent of the 
density profile. The critical temperature gradient, beyond which the toroidal ITG mode is unstable, depends most strongly on the electron density gradient, and is proportional to $\sqrt{T_{i} / T_{e}}$. Using the model of Ref. [19] to simulate the ion temperature at $100 \mathrm{~ms}$ intervals shows that (1) it falls approximately $45 \%$ low relative to the measured temperature prior to the pellet, (2) the simulated temperature stops evolving near the time the density profile recovers, and (3) the remaining increase due to $T_{i} / T_{e}$ is not sufficient to reproduce the longer time recovery of the measured temperature. Including $E_{r}$ shear as described in Sec. II, using a self-consistently calculated neoclassical radial electric field, reproduces the measured $T_{i}$ before, during, and after the perturbation.

It was observed in Ref. [13] that carbon pellets injected into TFTR supershots resulted in a rapid recovery of the density profile followed by a slower recovery of the energy confinement time. The carbon pellets had little effect on $\tau_{E}$ in L-Mode plasmas. Taken together with the present simulations, this provides a reason to invoke $E_{r}$ shear suppression as a feature distinguishing supershot from L-Mode plasmas. Preliminary investigation suggests that ELMy H-Mode plasmas on the DIII-D tokamak [30] respond similarly to relatively shallow deuterium pellet perturbations, with an extended temperature recovery, while L-Mode plasmas in DIII-D respond adiabatically on long timescales.

\section{III.B NONLINEAR SELF-REINFORCEMENT OF THE ION TEMPERATURE GRA- DIENT AND RADIAL ELECTRIC FIELD}

Here we consider a deuterium pellet perturbation of a very high performance supershot plasma produced under conditions similar to those of Ref. [31], taken from a campaign searching for toroidal Alfvén eigenmodes [32]. The discharge under consideration has little tangential torque input from its near-balanced neutral beams, although slightly counter-dominated. Following the perturbation, the density profile recovers entirely, while the ion temperature profile suffers a lasting factor of three degradation. Comparing the two times, when the density profile is the same, isolates the effect of $\nabla T_{i}$ on the impurity toroidal velocity. The strong ion temperature gradient produces a well structure or "notch," as explained using multispecies neoclassical calculations in Ref. [10]. This non-monotonic feature in the impurity toroidal velocity can result from neoclassical parallel heat friction of large hydrogenic parallel heat flows, which drag on the impurity in the counter-current direction. The parallel heat flow is driven by the the ion temperature gradient, producing a well-shape in the carbon toroidal rotation profile, most noticeable with balanced neutral beam injection. The notch feature is nevertheless consistent with hydrogenic toroidal velocity profiles which are monotonic (i.e., have shapes similar to the ion temperature profile $[23,24]$ ). If the radial transport of toroidal angular momentum is diffusive in nature, and torques such as those due to fast ion losses can be ignored, then the hydrogenic toroidal velocity profile must be monotonic, regardless of the torque deposition profile. This is supported by simulations of the central velocity evolution in off-axis beam modulation experiments [33] assuming diffusion only.

If the impurity poloidal rotation is small, as would be consistent with neoclassical calculations and available measurements (in much lower temperature plasmas), the measured carbon toroidal velocity profile closely resembles the radial electric field profile. Additional corrections due to density and temperature gradients further deepen the expected well in the radial electric field profile. Accordingly, stronger temperature gradients result in stronger $E_{r}$ shear, which may further suppress transport. Provided the resulting increase in the shearing rate meets or exceeds the increase in the maximum linear growth rate due to the increased temperature gradient, this illustrates a mechanism for positive self-reinforcement of the ion temperature with constant density profile. The closed-loop nonlinear simulations presented describe this self-reinforcement.

The global effects of a deuterium pellet perturbation are shown in Fig. 1. Two similar discharges are shown, one with a lithium pellet (\#75941) and one with a deuterium pellet (\#75936). The machine parameters for the deuterium pellet case are: neutral beam power $P_{b}=26.2 \mathrm{MW}$ (11 MW tritium), plasma current $I_{p}=2.0 \mathrm{MA}$, toroidal magnetic field $B_{\varphi}=5 \mathrm{~T}$, major $/$ minor radii $R_{0} / a=$ 2.52/0.87 meters, $T_{\text {cofr }}=-0.05$, line average electron density $\bar{n}_{e}=3.8 \times 10^{19} \mathrm{~m}^{-3}$, neutral beam 
injection energy $E_{b}=100 \mathrm{keV}$, and effective charge $Z_{\text {eff }}=2.6$, where $T_{\text {cofr }}=\left(T_{\text {co }}-T_{\text {ctr }}\right) /\left(T_{\text {co }}+T_{\text {ctr }}\right)$ and $T$ is the beam torque. Frame (a) shows the density profile peaking factor $n_{e}(0) /\left\langle n_{e}\right\rangle$ is affected similarly by the two types of pellet perturbations, which are quite similar despite entirely different injection systems. The pellet causes instantaneous flattening of the density profile, followed by a relaxation to the original peaked profile in roughly a particle confinement time. Often the density becomes slightly more peaked following the pellet if the beam power is held constant. A fortuitous loss of one neutral beam source (during the beam notch and pellet injection) allowed the electron density to recover a profile identical with that preceding the pellet. The energy confinement time in both cases suffers a lasting degradation to L-Mode values following the pellet, even after full recovery of the density profile. In these discharges, high power neutral beam heating was not sustained long enough to observe the eventual recovery of the stored energy. As shown in Fig. 1(e), the $H \alpha$ light, proportional to the edge hydrogenic particle flux, remains somewhat elevated upon recovery of the density profile. There is no lasting effect of the pellet on the carbon influx. Frame (d) shows the effect of the pellet on the electron density measured by fast laser interferometry, at chords intersecting the magnetic axis, the half-radius, and near the edge. The increment in density at the half-radius is slower than near the edge, indicating the pellet does not penetrate beyond the half-radius. The extra electrons near the outside increase the ionization rate of incoming beam neutrals, diminishing the beam penetration to the axis. This results in a decrease in the central density. The evolution of the ion temperature at the magnetic axis, half-radius, and near the edge is shown in Fig. 1(g). The central temperature drops by a factor of three, while the temperature at the half-radius drops by only a factor of two, and as shown in Frame (h), the temperature at the edge by only $20 \%$. The ion temperature does not show signs of recovery within the remaining $400 \mathrm{~ms}$ of neutral beam injection. The electron temperature profile is nearly unchanged at 3.87 seconds relative to 3.45 seconds. As shown in a later experiment, full ion reheat is attained by extending the beam duration.

The profiles before and after the perturbation are shown in Fig. 2. The ion temperature profile before the perturbation shows a very steep gradient near the one-third radius before the perturbation, and remains at L-Mode levels following density profile recovery, as shown near the end of the beam heating phase. The density profile recovers quickly and completely. Figure 2(c) shows the measured toroidal carbon velocity profiles at the same times before and after the pellet. The non-monotonic feature in the toroidal velocity profile is more pronounced and associated with the stronger temperature gradient prior to the pellet. The shearing rate shown in Frame (d) is calculated from the radial force balance equation using the measured toroidal velocity together with a poloidal velocity calculated numerically from the multispecies neoclassical parallel momentum balance using the TRV code [6, 10]. The peak radial electric field shearing rate is twice as large preceding the pellet. The linear growth rate of electrostatic toroidal drift modes, computed using the Kotschenreuther-Dorland-Liu GS2 linear gyrokinetic code [11] is also shown for comparison. The linear version of the GS2 code is a comprehensive gyrokinetic, initial value, stability code with physics content similar to and benchmarked against the FULL code [37]. These results show that the radial electric field shearing rate is of the order of the linear growth rate, and exceeds it over some fraction of the minor radius. Further, the linear growth rate and shearing rate vary together as functions of ion temperature in the region of steep ion temperature gradient [7].

The calculations using the GS2 code were carried out on a 64 processor SGI Origin 2000 parallel supercomputer using a recently developed, automated driver program written by the author. The driver program, GS2_PREP, reads TRANSP [22] data, prepares a GS2 namelist for each TRANSP radial zone, automatically runs GS2, and reports results. Scans over the product of perpendicular wavenumber and ion gyroradius generally resulted in maximum linear growth rates for $k_{\theta} \rho_{i} \simeq 0.18$, where $\rho_{i}=\sqrt{T_{i} / m_{i}} / \Omega_{i}$, and $m_{i}$ is the deuterium mass with $\Omega_{i}=m_{i} c / e B$ the deuterium cyclotron frequency. All growth rates shown were calculated with $k_{\theta} \rho_{i} \simeq 0.18$. Ideally, the perpendicular wavenumber which maximizes the linear growth rate should be found separately for each radius. For the runs presented, each beam energy component was treated as a separate species with a slowing-down distribution. 
The TRV code calculates neoclassical viscosity coefficients by numerically integrating over velocity space, solving the matrix equation relating the parallel and poloidal velocities of each species to the driving density and temperature gradients. The magnetic equilibrium was obtained from TRANSP, which showed excellent agreement with the measured diamagnetic flux and D-T neutron rate, indicating good consistency of the diagnostic dataset. The most recent version of TRV has been compared in detail with a recent version of the NCLASS code [34], resulting in near exact agreement once minor differences in friction coefficients and Coulomb logarithms were eliminated.

We attempted simulation of this discharge with the model of Ref. [19], using the TRV transport simulation code, but the model failed to converge, resulting in drastic overshoot of the ion temperature. The reason for this can be seen in Fig. 3, which shows the ratios of major radius to temperature gradient scale length from the model. If $R / L_{T}>R / L_{T}^{\text {crit }}$, where $R / L_{T}$ is evaluated from the measured temperature profile, then the toroidal ion temperature gradient (ITG) mode is unstable and dominant, while if $R / L_{T}>R / L_{T}^{\text {crit, } \mathrm{Z}}$, then the carbon branch of the toroidal ITG mode is unstable. Here Fig. 3(a) shows the stability criteria for the time 3.45 seconds preceding the pellet, and Fig. 3(b) shows the same criteria evaluated at 3.87 seconds following the pellet. At both times, the comparison suggests that the toroidal ITG mode is stable, while the measured temperature profile appears to be near marginal stability to the carbon toroidal ITG mode over much of the plasma cross-section. The part of the model describing the carbon branch appears to be less refined than for the main ITG branch, with the possible exception of its critical gradient. In addition, the resulting transport is quite sensitive to the $Z_{\text {eff }}$ profile, which is subject to large measurement uncertainties. For example, in TFTR, we have used a measurement of the carbon density profile obtained by charge exchange spectroscopy, normalized to fit the measured line-integrated visible Brehmstraahlung emission. The charge-exchange spectroscopy measurement depends on a calculation of the neutral beam penetration in the core and relevant cross-sections. On the other hand, $Z_{\text {eff }}$ profile data is available from a visible Brehmstraahlung chordal array, which in TFTR may be subject to reflected light. The $Z_{\text {eff }}$ profiles from the two measurements often differ significantly. Further, when the toroidal ITG mode is stable, trapped electron modes may be relatively more important, and are not well-described by the parameterization in the absence of ITG modes. The GS2 results show strongly growing modes with a phase velocity in the electron diamagnetic direction inside the one-quarter radius. In addition, ion modes not described by the parameterization appear to be increasingly unstable in the outer one quarter radius.

Nevertheless, evidence for the role of radial electric field shear can be found in Fig. 3 . In the high temperature phase, the parameterization suggests that the carbon toroidal ITG mode will be strongly unstable over the range 285 to $300 \mathrm{~cm}$ in major radius. Some form of turbulence suppression must be active in this region to allow deviation from marginal stability to this virulent transport mechanism, to attain the steep ion temperature gradients observed. On the other hand, at 3.87 seconds following the pellet, the same mode is predicted to be near marginal stability over most of the plasma cross-section. Therefore, radial electric field shear is less important at lower temperatures. Further, it is a distinguishing feature of supershot relative to degraded confinement.

\section{III.C SIMULATION OF TEMPERATURE SCAN PRODUCED BY DEUTERIUM PELLET PERTURBATION}

Among the last experiments performed on TFTR were several perturbation experiments motivated by these suggestions that pellet and helium puff perturbations could be used to study the effect of radial electric field shear on ion thermal confinement in supershot plasmas. Here we consider a supershot pellet perturbation experiment performed during this sequence. In contrast with \#75936, this experiment was performed with an extended period of neutral beam heating following the perturbation to look for possible long timescale recovery of the ion temperature, and to document the carbon poloidal rotation [35]. The plasma parameters for discharge \#104700 were $I_{p}=1.6 \mathrm{MA}$, $P_{b}=12.1 \mathrm{MW}, B_{\varphi}=4.8 \mathrm{~T}, R_{0} / a=2.51 / 0.86 \mathrm{~m} / \mathrm{m}, \bar{n}_{e}=2.4 \times 10^{19} \mathrm{~m}^{-3}, E_{b}=100 \mathrm{keV}$, and 
$T_{\text {cofr }}=0.25$ (co-dominated neutral beam injection). The eventual recovery of the stored energy is indeed observed on the timescale of several energy confinement times, twice that required for the recovery of the density profile. Our simulations of the ion temperature profile reproduce this slow recovery only when $E_{r}$ suppression is included. The longer timescale for energy recovery is shown to consist of two stages:

1. An initial period during which the density profile recovers entirely and the ion temperature increases, and during which radial electric field shear is unimportant, and

2. A roughly equal period following this in which radial electric field shear becomes important and leads to further increases in the ion temperature of up to $40 \%$.

The differing timescales for the recovery of density and temperature profiles in this experiment help delineate the effects of $E_{r}$ shear suppression and the influence of toroidal ITG mode marginal stability.

The evolution of the discharge \#104700, perturbed by a deuterium pellet $700 \mathrm{~ms}$ after the start of beam injection is shown in Fig. 5, together with a similar discharge \#104697 that did not have a perturbation. The density profile recovers in $300 \mathrm{~ms}$ to a attain slightly more peaked shape, matching the evolution of the unperturbed discharge. On the other hand, the global energy confinement time recovers on a timescale twice as long. The recovery is not complete, but matches the "rollover" in performance associated with a gradually increasing edge influx [36] as indicated by the unperturbed case (the unperturbed case had slightly higher heating power). The electron density evolution at the magnetic axis, the half-radius, and the edge shows that the pellet penetrated beyond the half-radius but not all the way to the magnetic axis. The central density increases slightly following the pellet and remains elevated for $800 \mathrm{~ms}$. Nearly identical behavior was obtained in a subsequent discharge \#104710.

The edge conditions are shown in Fig. 6. Following the pellet, the hydrogenic neutral influx settles to a slightly higher value than before the pellet, on the $300 \mathrm{~ms}$ timescale during which the density equilibrates. The carbon light is actually lower following the pellet. The edge ion temperature recovers on the longer $600 \mathrm{~ms}$ timescale on which the energy confinement time recovers. The central temperature also recovers on this timescale, but not completely. The increased hydrogenic recycling following the pellet is qualitatively consistent with the incomplete recovery of the energy confinement, and according to the empirical scaling $\tau_{E} \propto(H \alpha)^{-0.24}$ [29]. This higher neutral influx may be responsible for the slightly lower edge ion temperature, which may also prevent the core ion temperature from reaching its early values.

The calculated radial electric field and shearing rate profiles are flattened immediately following the pellet. The toroidal ITG linear growth rate and $E \times B$ shearing rate are driven in opposite directions during the perturbation. The shearing rate, evaluated using the TRV code from measured profiles, recovers on the slow $600 \mathrm{~ms}$ timescale to attain a $40 \%$ lower peak value.

We have found it necessary to modify the original growth rate model, first presented in Ref. [18]. The original model employed a growth rate parameterization of the form

$$
\gamma_{\text {lin }}^{\max }=\left(k_{\theta} \rho_{i}\right)_{\max } \frac{T_{e}}{T_{i}} \frac{v_{\text {thi }}}{R} \operatorname{Min}_{[\alpha=0.5,1.0]}\left(\frac{R}{L_{T}}-\frac{R}{L_{T}^{c r i t}}\right)^{\alpha},
$$

where $\left(k_{\theta} \rho_{i}\right)_{\max }=0.25$ and the thermal velocity $v_{\text {thi }}$ was evaluated for deuterium. Here we omit some small corrections for simplicity and have corrected the exponent according to well-known analytical theory of ITG modes. If the dominant source of transport is the carbon toroidal ITG mode, then $L_{T}^{\text {crit }}$, normally evaluated for deuterium, is replaced by the critical gradient for the carbon mode. In the original model, the thermal velocity was left unchanged when the carbon mode was dominant. In supershot plasmas, the deuterium branch is often stable over the inner $60 \%$ of the cross-section, dominating transport only in the outer L-Mode like region. The carbon branch appears to be near marginal stability in the inner half-radius, transitioning to the deuterium branch somewhere outside 
this. The critical gradient for deuterium is much larger than for carbon in supershot plasmas (but the reverse is generally true for L-Modes). In the original model this transition region resulted in large jumps in the model growth rate where the temperature gradient significantly exceeded the critical gradient for the carbon mode, but before it reached the critical gradient for the deuterium mode. Our initial comparisons with the GS2 code showed the growth rate from the model was more than twice as large as the more comprehensive calculation. We have corrected the problem by dividing $v_{\text {thi }}$ by $\sqrt{6}$ whenever the carbon mode dominates transport. In addition, we have changed $\left(k_{\theta} \rho_{i}\right)_{\max }$ from 0.25 to 0.18 , the same value used in the GS2 calculations. The revised model growth rate compares quite well with the GS2 code, as shown in Fig. 4. In this case, the phase velocity of the fastest growing electrostatic mode is in the ion diamagnetic direction over the entire cross-section, roughly consistent with the assumed relative unimportance of trapped electron destabilization. Note the GS2 code includes trapped electrons. A strong ion mode is apparent in the GS2 growth rate near the edge, not accounted for in the parameterization. For this reason the simulations are restricted to $r / a \lesssim 0.85$ as in Ref. [19].

We now turn to simulations of the slow ion temperature evolution using the model of Refs. $[6,7]$ with self-consistent neoclassical radial electric field. Figure 5(c) shows the results of the original model of Ref. [19] and the extended model with self-consistent radial electric field. The calculations are shown at $r / a=0.2$ where the magnetic shear is within the range of validity for the model of Ref. [19]. Without $E_{r}$ shear, the simulated core temperature is $35 \%$ lower, strongly underestimating the ion temperature preceding the pellet. During the initial density profile recovery, $E_{r}$ shear is not necessary to reproduce the measured temperatures. However, shortly after the density profile stops evolving, the ion temperature from the simulation without $E_{r}$ shear saturates, and does not follow the continued reheat at constant density. When $E_{r}$ shear is included and calculated simultaneously with the ion temperature, the simulations reproduce this slow reheat phase.

Figure 7 shows the details of a simulation of \#104700n01 at 3.10 seconds, using the fully revised model with $\alpha_{E}=0.5$. Frame (a) shows that the simulated ion temperatures increase $60 \%$ at the magnetic axis when $E_{r}$ shear is turned on. Frame (b) compares the simulated ion temperature gradient and the critical gradients for the deuterium and carbon toroidal ITG modes, both with (solid) and without (dashed) $E_{r}$ shear. Here it is apparent that the $60 \%$ increase in temperature associated with $E_{r}$ shear does not significantly affect the critical gradients. The proximity to marginal stability is typical of supershots we have examined. Inside $r / a<0.40$, and without $E_{r}$ shear, the simulated temperature gradient is marginally stable to the carbon mode. There is a transition region $0.40<r / a<0.55$ over which the carbon ITG mode becomes progressively more unstable until the deuterium mode is destabilized and thereafter dominates transport. It is apparent that the carbon mode critical gradient lacks the pronounced radial structure required to match the steep measured ion temperature gradient in the core, if the measured ion temperature profile were marginally stable to the carbon mode. Including $E_{r}$ shear allows the simulated ion temperature gradient to more than double relative to marginal stability, without virulent transport, reaching measured values. Frame (c) shows the linear growth rate and self-consistent neoclassical $E_{r}$ shearing rate from the same simulation. Their near-equality in the inner half-radius supports our previous findings [7] that the supershot core is characterized by the criterion $\alpha_{E} \omega_{E \times B} \sim \gamma_{\text {lin }}^{\max }$.

In summary, a relatively complete description of transport due to toroidal ion temperature gradient driven turbulence, which well reproduces the temperature profiles of L-Mode plasmas in TFTR, was insufficient to describe the reheat to very high core temperatures in supershot plasmas. Including the effect of turbulence suppression by a radial electric field, with spatial and temporal scales large compared to those of the turbulence, resulted in agreement with measured temperatures. The increasingly important role of $E_{r}$ shear as the ion temperature increases is conveniently illustrated in this temperature scan produced during the reheat following a deuterium pellet perturbation.

Finally, the version of the TRV code used here was not fully time-dependent, so one may ask whether this influenced the results. Because measured density profiles are used, the time-dependence arises through the thermal inertia term $(3 / 2) \partial_{t}\left(n_{i} T_{i}\right)$. This term is included in the ion power balance 
as read from TRANSP; it is not simulated. While at first this treatment may appear to unfairly influence the simulation with information from the $T_{i}$ measurement, this is not a significant effect during the slow recovery following the pellet. The term $(3 / 2) \partial_{t}\left(n_{i} T_{i}\right)$ as a fraction of the total power density conducted and convected by the ions, has been evaluated from TRANSP in Ref. [6]. During the time 3.4 to 3.7 seconds, the fraction increases from $-20 \%$ to $+10 \%$, and diminishes thereafter. Therefore, using the measured ion temperature to evaluate the thermal inertia term in the power balance does not influence the evolution of the calculated ion temperature strongly.

We note the simulations in Fig. 5 were carried out with an older implementation of the model. After several corrections, including those discussed above, the revised model gives similar results, but with $\alpha_{E}=0.5$ instead of $\alpha_{E}=1.0$.

\section{CONCLUSIONS}

We have found experimental signatures to illustrate a mechanism for positive self-reinforcement of the ion temperature gradient in TFTR supershot plasmas through $E_{r}$ shear suppression. Investigating perturbations of supershot plasmas by deuterium pellets reveals that the pellets produce temperature scans lasting several confinement times, following rapid recovery of the density profile. By simulating the reheat following such pellet perturbations, we demonstrated that $E_{r}$ shear becomes more important as the ion temperature increases and is necessary to reproduce this extended reheat phase at constant density. The same reheat phase is not qualitatively or quantitatively described by a relatively comprehensive parameterization of transport driven by toroidal ITG turbulence [19] without including $E_{r}$ shear. This provides a distinct qualitative reason to invoke $E_{r}$ shear suppression to explain improved confinement in TFTR supershot plasmas, despite the absence of an apparent transition to improved confinement. This is of particular value because the anticipated effect of $E_{r}$ shear per se would otherwise be difficult to distinguish qualitatively from other stabilizing influences on toroidal ITG modes, such as peaked density profiles.

This approach has led to a new understanding of disparate features of enhanced ion thermal confinement in TFTR plasmas $[6,7]$. The results are also consistent with a criterion for nearcomplete turbulence suppression in the inner half-radius described by $\alpha_{E}\left|\omega_{E \times B}\right| \simeq \gamma_{\text {lin }}^{\max }$, from which the ion temperature profile can be obtained. This self-regulation occurs on much larger spatial and temporal scales than the fine-scale, fluctuating zonal flows known to be important to the nonlinear turbulence saturation level [38, 39, 40, 41, 42].

Finally, we remark that to reproduce the favorable confinement scalings of supershot plasmas, it is necessary that the radial electric field have a favorable ion temperature dependence. Very preliminary investigations of the finite lifetime effect [9] suggest that the carbon poloidal rotation may be slightly stronger in the electron diamagnetic direction than neoclassical estimates. This discrepancy may be accounted for by the poloidal torque driven by the radially inward return current of thermal ions which maintain charge neutrality in the presence of lost beam ions (i.e., trapped beam ions lost after their first turning point or those lost due to ripple or Alfvén eigenmodes). The radial electric field resulting from such a return current is given roughly by $E_{r} \simeq\left(\Omega_{i} / \mu_{i}\right)\left(u_{i r} / c\right) B_{\varphi}$, where $\Omega_{i}=Z_{i} e B_{\varphi} / m_{i} c$ is the ion cyclotron frequency, $\mu_{i} \propto n_{i} / T_{i}^{3 / 2}$ is the neoclassical poloidal flow damping rate, $c$ is the speed of light, $u_{i r}$ is the (negative) radial velocity of returning ions, and $B_{\varphi}$ is the toroidal magnetic field. This produces a negative radial electric field with a strongly favorable ion temperature dependence.

It is a pleasure to thank D. C. McCune and C. Ludescher for TRANSP development, and Drs. J. Hosea and R. Goldston for support. This work was supported by U.S. DoE Contract DE-AC0276-CH03073. 


\section{A EMPIRICAL SCALING FOR APPARENT CARBON POLOIDAL ROTATION IN TFTR SUPERSHOT PLASMAS}

In the final two months of operation of TFTR, a new poloidal rotation diagnostic was in operation [43, 35]. Analysis of data for supershot plasmas revealed a significant discrepancy with the neoclassical calculation of the carbon poloidal velocity. The discrepancy appears as a large positive offset, nonuniform in radius, with measured poloidal velocities greatly in excess of neoclassical estimates $[10,34]$. However, following the presentation of the results in this Appendix, a new effect involving the finite lifetime of the observed carbon 5+ VIII to VII line and the energy dependence of the excitation cross-section, in magnetized plasmas, was discovered [9]. Preliminary investigation shows this effect is large enough to change the sign of the apparent carbon poloidal velocity, changing the interpretation of spectroscopic poloidal rotation measurements at high temperatures. Therefore, we have reverted to a neoclassical calculation of the carbon poloidal velocity as in our prior model pending quantitative analysis of this new effect. In the following paragraphs, the poloidal rotation obtained from spectroscopy without including the finite lifetime effect is referred to as the "apparent" rotation.

The discrepancy between the apparent and neoclassical rotation is conveniently illustrated in an experiment which changed the tangental neutral beam torque from pure co-injection to balanced injection at constant power in a supershot plasma, shown in Fig. 8. While the toroidal velocity decreases almost to zero when the torque is removed, the measured poloidal velocity appears to more closely follow the ion temperature decrease. The neoclassical value [21] is calculated from a code which evaluates the viscosities numerically by integration over velocity space and solves the two-species stress-friction relations for poloidal velocities and heat flows [10]. Similar conclusions are found using an independent code [34]. The measured radial electric field $E_{r}$ shows a positive offset relative to that inferred using the neoclassical poloidal rotation.

Figure 9 shows the radial profiles before and after the torque change. With pure co-injection, the discrepancy in poloidal rotations produces a qualitative, rather than quantitative difference in the $E_{r}$ profile. However, for balanced injection, the carbon poloidal velocity $u_{\theta C}$ is the dominant term in the radial force balance equation (the pressure gradient term is weighted by $1 / Z$ ), as shown in Frame (c). In this particular case, the measured and neoclassical $E_{r}$ profiles have opposite signs. However, because the shearing rate is independent of the sign, the shearing rate profiles are qualitatively similar, both reaching a maximum just inside the quarter-radius. It is worth mentioning that the transport model using a neoclassical radial electric field produced similar results for the ion temperature because $E_{r}^{\text {neo }} \simeq-T_{i} / e r_{n}$, where $r_{n}^{-1}=-d \ln n_{i} / d r$, so that the shearing rate increased with $T_{i}[6,7]$. In the following paragraphs, we show that the same is true when the apparent measured poloidal rotation is used to infer $E_{r}$, preserving the nonlinear behavior of the model, which is important to reproduce the confinement trends. However, because the shearing rate associated with the apparent poloidal rotation was larger, it was necessary to reduce $\alpha_{E}$ from unity to 0.5 .

The apparent measured carbon poloidal rotation in TFTR supershots can be described with a simple linear fit to the measured ion temperature given by $u_{\theta C}(r) \mathrm{km} / \mathrm{s}=\left\{u_{\theta 0}+\alpha_{\theta T}\left(T_{i}(r)-\right.\right.$ $\left.\left.T_{i}(a)\right)\right\}(\mathrm{keV})$, where $u_{\theta 0}=0.182, \alpha_{\theta T}=1.43, r$ is the local minor radius and the plasma edge lies at $r=a$. The empirical scaling is shown in Fig. 10, and is based on time-dependent data from eleven supershot discharges and one L-Mode discharge. The majority of the discharges had similar operating parameters $(4.8 \mathrm{~T}, 1.6 \mathrm{MA}, 12.1-13.5 \mathrm{MW}$, major radius $251 \mathrm{~cm}$, minor radius $86 \mathrm{~cm}$, positive magnetic shear, and circular cross-section). The fact that the points near the edge do not fit the scaling is particularly significant in reference to the finite lifetime effect. These points correspond to intrinsic line emission rather than lines excited by charge exchange with beam neutrals.

Figure 11 shows temporal evolution of the fit follows the apparent measured poloidal rotation, shown near the one-third radius, during perturbation experiments in supershot plasmas. The radial profiles before and after the perturbations are shown in Fig. 12. We emphasize that, while the 
empirical scaling for $u_{\theta C}$ seems to reproduce apparent measured profiles in TFTR supershots, it is based on a relatively limited dataset which may limit its generality.

A simpler fit, $u_{\theta C}(\mathrm{~km} / \mathrm{s}) \simeq T_{i}(\mathrm{keV})$ has also been given [44]. Other dependences are apparent especially near the edge, where there is little correlation of the poloidal velocity with ion temperature. We examined other possibilities as well. For example, the correlation with the energy confinement time $\tau_{E}$ initially appeared promising, but was broken in the torque change experiments. Another possible expectation $\Phi \propto T_{e}$, where $\Phi$ is the electrostatic potential and $T_{e}$ the electron temperature, formulated under the assumption $T_{i}=0$ [38], was uncorrelated. Relaxing the latter assumption might lead to $\Phi \propto T_{i}$, implying $u_{\theta C} \propto \nabla T_{i} / B$, which was a worse fit than $u_{\theta C} \propto T_{i}$, especially in high temperature cases.

An empirical scaling for the carbon poloidal rotation, roughly proportional to the ion temperature, has been shown to reproduce the apparent measured poloidal rotation data. In the original presentation, we made use of the empirical scaling for $u_{\theta C}$ to simulate ion temperature profiles in supershots for which no poloidal rotation data was available. To compensate for the larger measured poloidal rotation relative to our prior neoclassical estimate, the coefficient $\alpha_{E}$ had to be reduced from unity to 0.5 .

\section{REFERENCES}

[1] K. Burrell, Phys. Plasmas 4, 1499 (1997).

[2] M. Bell, R. E. Bell, P. C. Efthimion, D. R. Ernst, E. D. Fredrickson, F. M. Levinton, J. Manickam, E. Mazzucato, G. L. Schmidt, E. J. Synkowski, M. C. Zarnstorff, and the TFTR Group, Plasma Phys. Control. Fusion 41, A719 (1999), Invited Paper, Proc. 25th European Physical Soc. Conference on Controlled Fusion and Plasma Physics.

[3] R. J. Hawryluk, Rev. Mod. Phys. 70, 537 (1998).

[4] R. Hawryluk, S. Batha, W. Blanchard, M. Beer, M. G. Bell, R. E. Bell, H. Berk, et al., Phys. Plasmas 5, 1577 (1998).

[5] R. J. Hawryluk, Phil. Trans. R. Soc. Lond. A 357, 443 (1999).

[6] D. R. Ernst, Momentum Transport, Radial Electric Field, and Ion Thermal Energy Confinement in Very High Temperature Plasmas, PhD thesis, Physics Dept., Mass. Inst. of Technology, 1997.

[7] D. R. Ernst, B. Coppi, S. D. Scott, M. Porkolab, and the TFTR Group, Phys. Rev. Lett 81, $2454(1998)$.

[8] D. R. Ernst, S. Batha, M. Beer, M. G. Bell, R. E. Bell, R. V. Budny, B. Coppi, W. M. Dorland, P. C. Efthimion, T. S. Hahm, G. W. Hammett, R. J. Hawryluk, K. W. Hill, M. Kotschenreuther, F. M. Levinton, Z. Lin, D. K. Mansfield, D. R. Mikkelsen, R. Nazikian, M. Porkolab, G. Rewoldt, S. D. Scott, E. J. Synakowski, M. C. Zarnstorff, and the TFTR Group, in Plasma Physics and Controlled Nuclear Fusion Research, 1998, Int'l. Atomic Energy Agency, Vienna, 1998, Paper IAEA-CN-69/EXP1/14(R).

[9] M. C. Zarnstorff, Private communication, Princeton Plasma Physics Laboratory (1998).

[10] D. R. Ernst, M. G. Bell, R. E. Bell, C. E. Bush, Z. Chang, E. Fredrickson, L. R. Grisham, K. W. Hill, D. L. Jassby, D. K. Mansfield, D. C. McCune, H. K. Park, A. T. Ramsey, S. D. Scott, J. D. Strachan, E. J. Synakowski, G. Taylor, M. Thompson, and R. M. Wieland, Phys. Plasmas 5, 665 (1998).

[11] M. Kotschenreuther and G. Rewoldt, Comp. Phys. Comm., 88, 128 (1995). 
[12] P. C. Efthimion, S. von Goeler, W. A. Houlberg, E. J. Synakowski, M. C. Zarnstorff, S. H. Batha, R. E. Bell, M. Bitter, C. E. Bush, et al., Phys. Plasmas 5, 1832 (1998).

[13] R. Hulse, A. Cavallo, D. Johnson, H. Park, A. Ramsey, G. Schmidt, B. Stratton, E. Synakowski, G. Taylor, J. Terry, B. Howell, and E. Marmar, Perturbative transport studies of neutral beam heated TFTR plasmas using carbon pellet injection, in Proceedings of the 17th European Conference on Controlled Fusion and Plasma Heating, Amsterdam, 1990, volume 1, pp. 46-49, European Physical Society, Petit-Lancy, Switzerland, 1990.

[14] E. Fredrickson, K. McGuire, R. J. Goldston, S. Hiroe, M. C. Zarnstorff, M. Bell, N. Bretz, J. D. Callen, P. C. Efthimion, B. Grek, D. Johnson, D. McCune, G. Taylor, N. Sauthoff, F. Stauffer, and R. Wieland, Nucl. Fusion 27, 1897 (1987).

[15] R. E. Waltz, G. D. Kerbel, and J. Milovich, Phys. Plasmas 1, 2229 (1994).

[16] T. S. Hahm and K. Burrell, Phys. Plasmas 2, 1648 (1995).

[17] H. Biglari, P. H. Diamond, and P. W. Terry, Phys. Fluids B 2, 1 (1990).

[18] W. Dorland, M. Kotschenreuther, Q. Liu, M. Beer, and G. Hammett, in Proc. of the Joint Varenna-Lausanne Int. Workshop on Theory of Fusion Plasmas (August 1996), Varenna, Italy 1996 (Editrice Compositori, Bologna, Italy), p. 185.

[19] M. Kotschenreuther, W. Dorland, M. Beer, and G. W. Hammett, Phys. Plasmas 2, 2381 (1995).

[20] C. S. Chang and F. L. Hinton, Phys. Fluids 29, 3314 (1986).

[21] S. P. Hirshman and D. J. Sigmar, Nucl. Fusion 21, 1079 (1981).

[22] R. J. Goldston, D. C. McCune, H. H. Towner, S. L. Davis, R. J. Hawryluk, and G. L. Schmidt, J. Comp. Phys. 43, 61 (1981).

[23] S. Scott, P. Diamond, R. Fonck, R. Goldston, R. Howell, K. Jaehnig, G. Schilling, E. Synakowski, M. Zarnstorff, C. Bush, E. Fredrickson, K. Hill, A. Janos, D. Mansfield, D. Owens, H. Park, G. Pautasso, A. Ramsey, J. Schivell, G. Tait, W. Tang, and G. Taylor, Phys. Rev. Lett. 64, 531 (1990).

[24] S. Scott, V. Arunasalam, C. W. Barnes, M. Bell, M. Bitter, et al., Phys. Fluids B 2, 1300 (1990).

[25] H. Park, Plasma Phys. and Contr. Fusion 31, 2039 (1989).

[26] H. K. Park, M. G. Bell, W. M. Tang, G. Taylor, and M. Yamada, Nucl. Fusion 34, 1272 (1994).

[27] H. K. Park, M. G. Bell, M. Yamada, K. McGuire, S. Sabbagh, R. V. Budny, R. Wieland, S. Ishida, T. Nishitani, Y. Kawano, Y. Y. Kamada, T. Fujita, M. Mori, Y. Koide, H. Shirai, M. Kikuchi, and the JT-60 Team, in Proceedings of the Fifteenth International Atomic Energy Agency Conference on Plasma Physics and Controlled Nuclear Fusion Research 1994, (September 26-October 1, 1994, Seville, Spain), pub. Int'l. Atomic Energy Agency, Vienna, 1995., volume 1, p. 211.

[28] H. K. Park, S. A. Sabbagh, S. Batha, M. Bell, R. V. Budny, C. Bush, Z. Chang, D. Johnson, D. K. Mansfield, D. McCune, K. M. McGuire, R. Nazikian, C. Skinner, R. Wieland, M. Yamada, and K. M. Young, Phys. Plasmas 4, 1699 (1997).

[29] J. D. Strachan, M. Bell, A. Janos, S. Kaye, S. Kilpatrick, D. Manos, D. Mansfield, D. Mueller, K. Owens, C. S. Pitcher, J. Snipes, and J. Timberlake, J. Nucl. Mat 196-198, 28 (1992). 
[30] J. L. Luxon, R. Anderson, F. Batty, C. B. Baxi, G. Bramson, N. H. Brooks, B. Brown, et al., in Plasma Physics and Controlled Nuclear Fusion Research, 1986, Int'l. Atomic Energy Agency, Vienna, 1987, 1986, Vol. I, p. 159.

[31] J. D. Strachan, H. Adler, P. Alling, C. Ancher, H. Anderson, J. L. Anderson, D. Ashcroft, C. W. Barnes, G. Barnes, S. Batha, M. G. Bell, R. Bell, M. Bitter, W. Blanchard, N. L. Bretz, R. Budny, C. E. Bush, R. Camp, M. Caorlin, S. Cauffman, Z. Chang, C. Z. Cheng, J. Collins, G. Coward, D. S. Darrow, J. DeLooper, H. Duong, L. Dudek, R. Durst, P. C. Efthimion, D. Ernst, R. Fisher, R. J. Fonck, E. Fredrickson, N. Fromm, G. Y. Fu, H. P. Furth, C. Gentile, N. Gorelenkov, B. Grek, L. R. Grisham, G. Hammett, G. R. Hanson, R. J. Hawryluk, W. Heidbrink, H. W. Herrmann, K. W. Hill, J. Hosea, H. Hsuan, A. Janos, D. L. Jassby, F. C. Jobes, D. W. Johnson, L. C. Johnson, J. Kamperschroer, H. Kugel, N. T. Lam, P. H. LaMarche, M. Lenard, F. M. Levinton, J. Machuzak, D. Mansfield, A. Martin, E. Mazzucato, R. Majeski, M. J. Loughlin, B. LeBlanc, E. Marmar, J. McChesney, B. McCormack, D. C. McCune, K. M. McGuire, G. McKee, D. M. Meade, S. S. Medley, D. R. Mikkelsen, D. Mueller, M. Murakami, A. Nagy, R. Nazikian, R. Newman, T. Nishitani, M. Norris, T. O'Connor, M. Oldaker, M. Oskabe, D. K. Owens, H. Park, S. F. Paul, G. Pearson, E. Perry, M. Petrov, C. K. Phillips, S. Pitcher, A. T. Ramsey, D. A. Rasmussen, M. H. Redi, D. Roberts, J. Rogers, R. Rossmassler, A. L. Roquemore, E. Ruskov, S. A. Sabbagh, M. Sasao, G. Schilling, J. Schivell, G. L. Schmidt, S. D. Scott, R. Sissingh, C. H. Skinner, J. A. Snipes, J. Stevens, T. Stevensen, B. C. Stratton, E. Synakowski, W. Tang, G. Taylor, J. L. Terry, M. E. Thompson, M. Tuszewski, C. Vannoy, A. von Halle, S. von Goeler, D. Voorhes, R. T. Walters, R. Wieland, J. B. Wilgen, M. Williams, J. R. Wilson, K. L. Wong, G. A. Wurden, M. Yamada, K. M. Young, M. C. Zarnstorff, and S. J. Zweben, Phys. Rev. Lett. 72, 3526 (1994).

[32] S. J. Zweben, R. V. Budny, C. Z. Cheng, E. D. Fredrickson, G. Y. Fu, D. R. Mikkelsen, G. L. Schmidt, S. D. Scott, D. A. Spong, C. E. Bush, Z. Chang, D. S. Darrow, R. J. Fonck, L. R. Grisham, E. Mazzucato, R. Nazikian, D. K. Owens, H. K. Park, S. F. Paul, J. F. Schivell, J. D. Strachan, E. J. Synakowski, G. Taylor, and K. M. Young, Nucl. Fusion 36, 987 (1996).

[33] R. Goldston, V. Arunasalam, M. Bell, M. Bitter, W. Blanchard, et al., Energy confinement and profile consistency in TFTR, in Plasma Physics and Controlled Nuclear Fusion Research 1986, volume 1, pp. 75-87, IAEA,Vienna, 1987.

[34] W. Houlberg, K. C. Shaing, S. P. Hirshman, and M. C. Zarnstorff, Phys. Plasmas 4, 3230 (1997).

[35] R. E. Bell, Rev. Sci. Instrum. 68, 1273 (1997).

[36] J. Strachan, Nucl. Fusion 34, 1017 (1994).

[37] G. Rewoldt, W. M. Tang, and R. J. Hastie, Phys. Fluids 30, 807 (1987), First publication of "FULL" code.

[38] A. Hasegawa and M. Wakatani, Phys. Rev. Lett. 59, 1581 (1987).

[39] M. N. Rosenbluth and F. L. Hinton, Phys. Rev. Lett. 80, 724 (1998).

[40] M. A. Beer and G. W. Hammett, in Proc. of the Joint Varenna-Lausanne Int. Workshop on Theory of Fusion Plasmas (August 1998), Varenna, Italy 1998 (Editrice Compositori, Bologna, Italy, 1999).

[41] P. Diamond, M. N. Rosenbluth, F. L. Hinton, M. Malkov, J. Fleischer, and A. Smolyakov, in Plasma Physics and Controlled Nuclear Fusion Research, 1998, Int'l. Atomic Energy Agency, Vienna, 1998, Paper IAEA-CN-69/TH3/1. 
[42] A. Dimits, B. I. Cohen, N. Mattor, W. M. Nevins, and D. E. Shumaker, in Plasma Physics and Controlled Nuclear Fusion Research, 1998, Int'l. Atomic Energy Agency, Vienna, 1998, Paper IAEA-CN-69/TH1/1.

[43] R. E. Bell, Phys. Rev. Lett. 81, 1429 (1998).

[44] R. E. Bell, S. H. Batha, M. G. Bell, R. V. Budny, D. R. Ernst, D. W. Johnson, B. LeBlanc, F. Levinton, H. Park, A. T. Ramsey, E. J. Synakowski, G. Taylor, and M. C. Zarnstorff, in Plasma Physics and Controlled Nuclear Fusion Research, 1998, Int'l. Atomic Energy Agency, Vienna, 1999, Paper IAEA-CN-69/EXP1/13(R). 

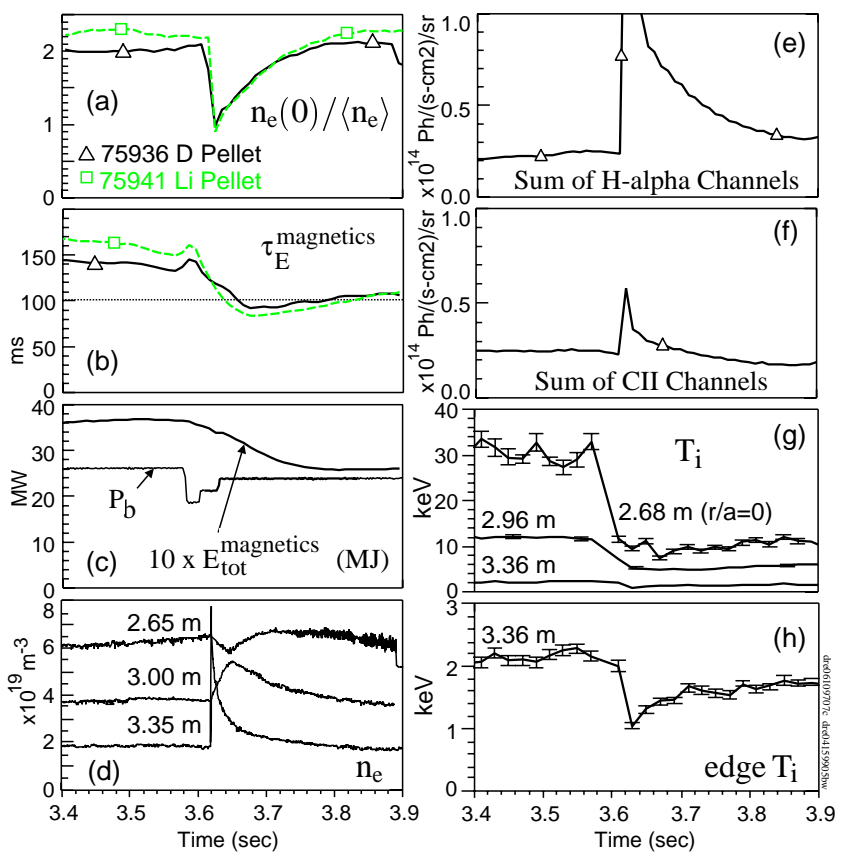

FIG. 1. Effect of pellet perturbations in supershot plasmas. (a) Electron density peakedness recovers rapidly, after which the electron density profile closely resembles the initial one. Both deuterium and lithium pellet perturbations are shown. (b) Global energy confinement time remains at L-Mode levels even after density profile fully recovers. (c) Total stored energy and neutral beam power for the deuterium pellet case, showing the nonadiabatic character of the perturbation. (d) Density chords near the magnetic axis, half-radius, and edge for the deuterium pellet case. (e) Balmer line emission for the D pellet case does not fully relax before the end of beams. (f) Carbon light fully relaxes. (g) Measured ion temperature near magnetic axis, half-radius, and edge for the deuterium pellet case. (h) Edge temperature recovers on timescale of density recovery. 


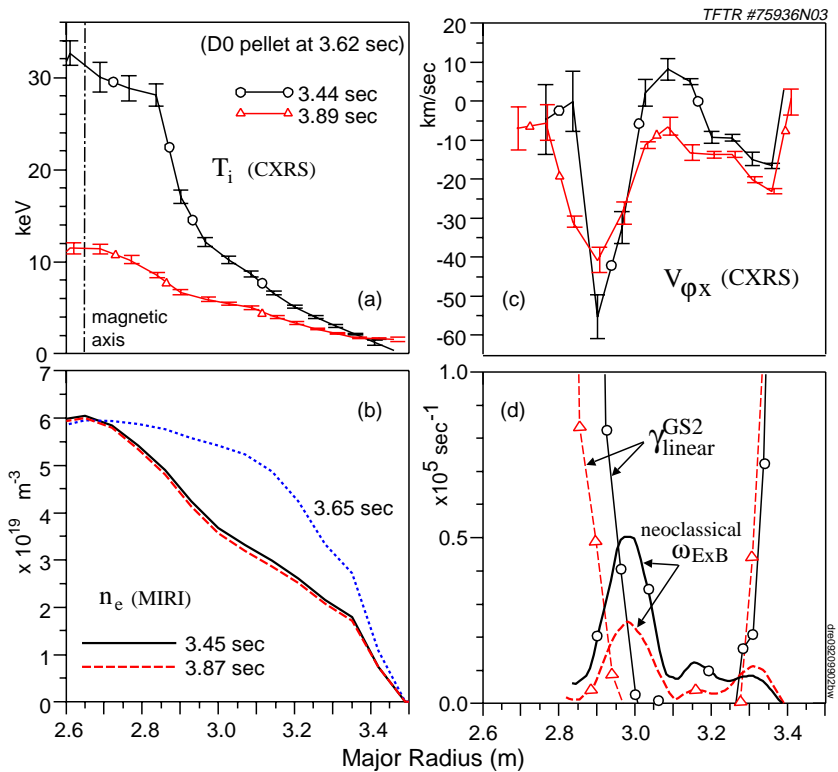

FIG. 2. Profiles before and after deuterium pellet perturbation. (a) The measured ion temperature profiles before and after the perturbation. (b) Measured density profiles before, during, and after perturbation. (c) The measured carbon toroidal velocity profile before the perturbation has a large notch at the radius of steepest ion temperature gradient. The feature is less pronounced at lower temperatures. (d) The peak $E \times B$ shearing rate calculated assuming neoclassical poloidal rotation.
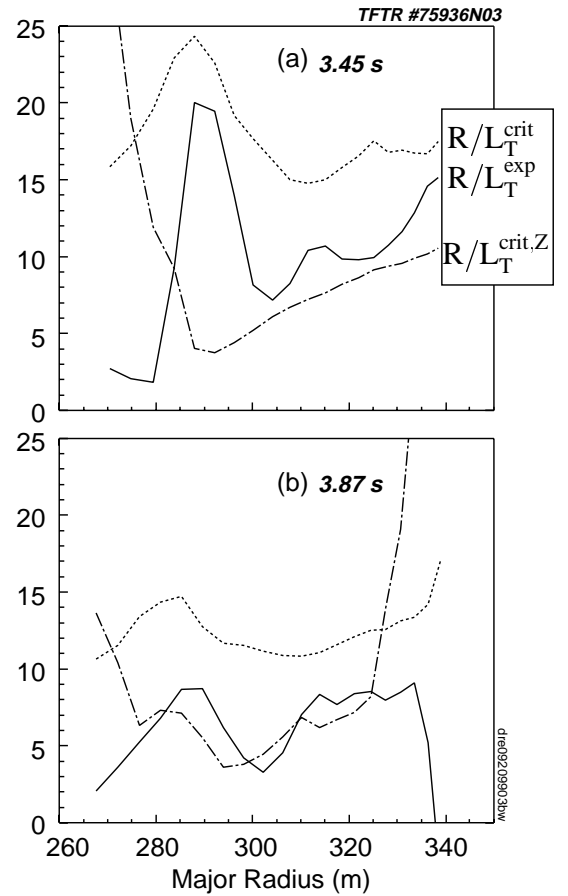

FIG. 3. Linear stability of toroidal ITG modes from the IFS-PPPL parameterization. (a) Before the pellet. (b) After the pellet. 


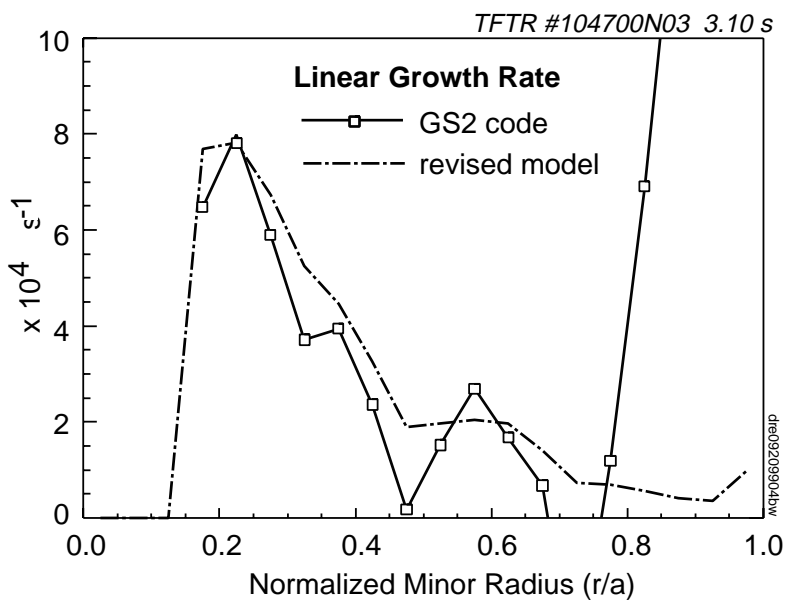

FIG. 4. Comparison of linear growth rates from the GS2 gyrokinetic code and the revised model growth rate parameterization for \#104700N03 at 3.1 seconds. The carbon toroidal ITG mode is dominant over most of the plasma cross-section. 


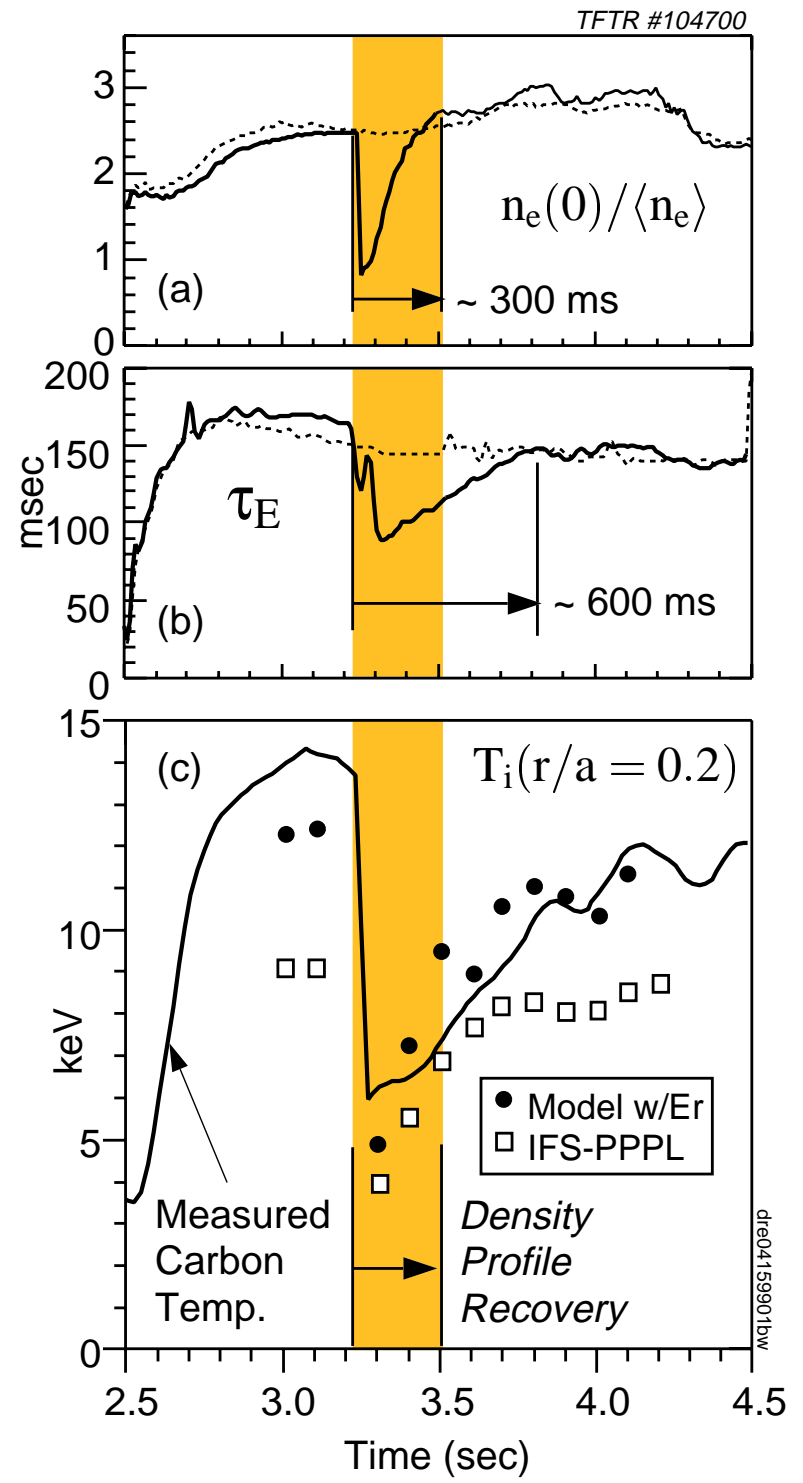

FIG. 5. Simulation of deuterium pellet perturbation $700 \mathrm{~ms}$ following the start of neutral beam injection (\#104700). (a) The electron density profile recovers on a timescale $300 \mathrm{~ms}$, and is more peaked after the pellet. A reference discharge is shown for comparison, illustrating temporal changes that occur independent of the pellet. (b) The global energy confinement time recovers much more slowly than the density profile, on a timescale approximately twice as long. (c) Measured and simulated ion temperatures at $r / a=0.2$ with and without $E_{r}$ shear. 


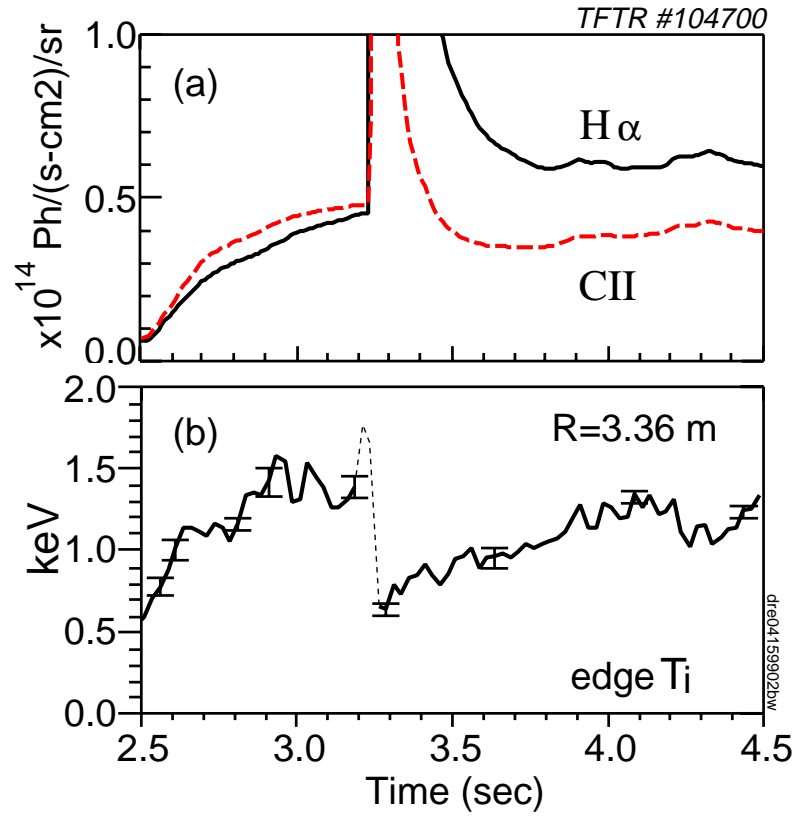

FIG. 6. Edge conditions during deuterium pellet pellet perturbation in \#104700. (a) $H \alpha$ and CII light. The increase in $H \alpha$ light projects to a $-4 \%$ change in $\tau_{E}$, but the observed change is $-18 \%$. (b) The change in edge ion temperature is of order $-13 \%$ upon recovery. The change in central ion temperature upon recovery is $-13 \%$ as well. 

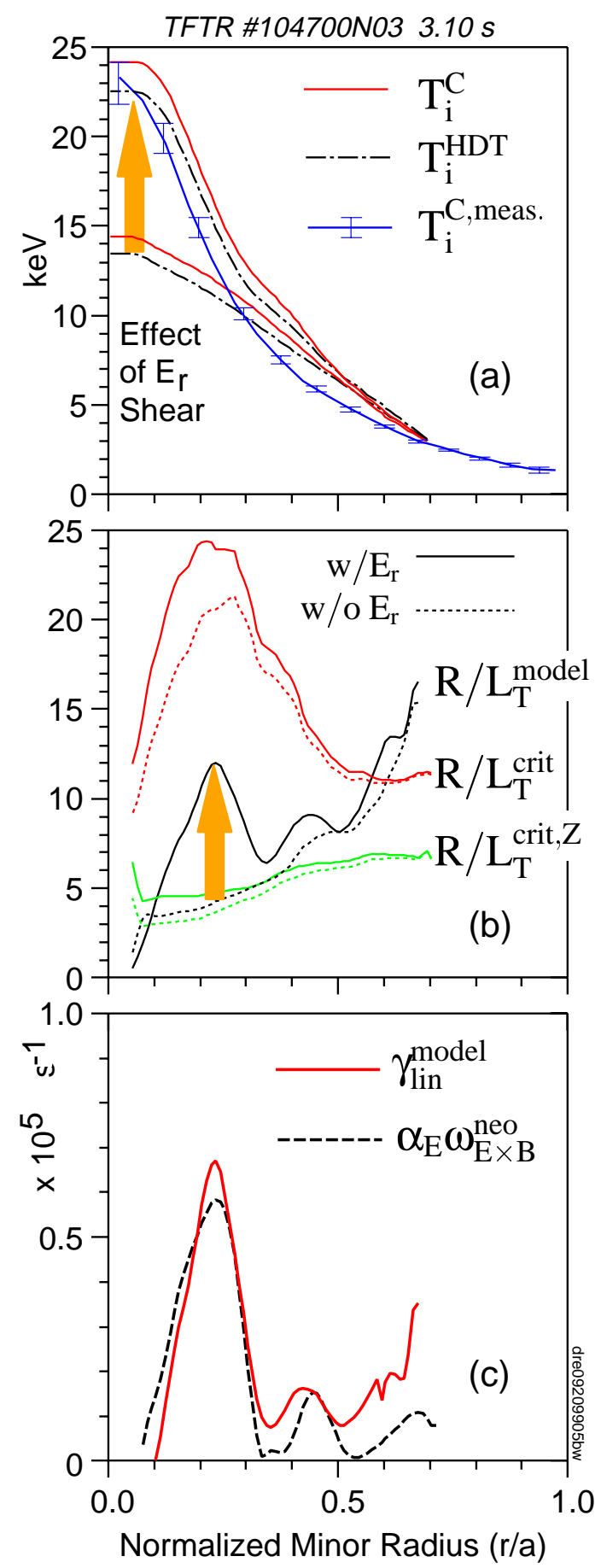

FIG. 7. Simulation of \#104700N01 at 3.10 seconds. (a) Simulated ion temperatures for carbon (solid) and hydrogenic species (dot-dash) compared with the measured carbon ion temperature (error bars). Results are shown both with and without $E_{r}$ shear. (b) Simulated ion temperature gradient compared with critical gradients for deuterium and carbon toroidal ITG modes. Results shown with $E_{r}$ shear (solid) and without (dashed). (c) Self-consistent linear growth rate $\left(\gamma_{\text {lin }}^{\max }\right)$ and neoclassical shearing rate $\left(\alpha_{E} \omega_{E \times B}^{\text {neo }}\right)$ from the same simulation. 

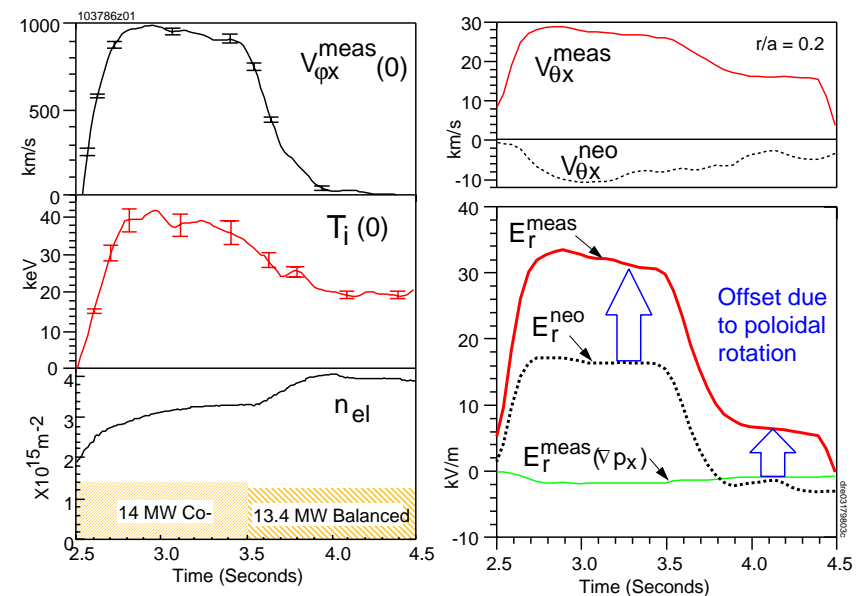

FIG. 8. Experiment changing neutral beam torque from co- to balanced at constant heating power. (a) Measured central carbon toroidal velocity. (b) Measured central carbon temperature. (c) Measured line integral electron density. (d) Apparent measured carbon poloidal velocity near its radial maximum, together with that from a numerical neoclassical calculation using a $4 \times 4$ viscosity matrix and numerically integrated viscosities. (e) Radial electric field calculated using neoclassical poloidal velocity compared with that using apparent measured poloidal velocity.
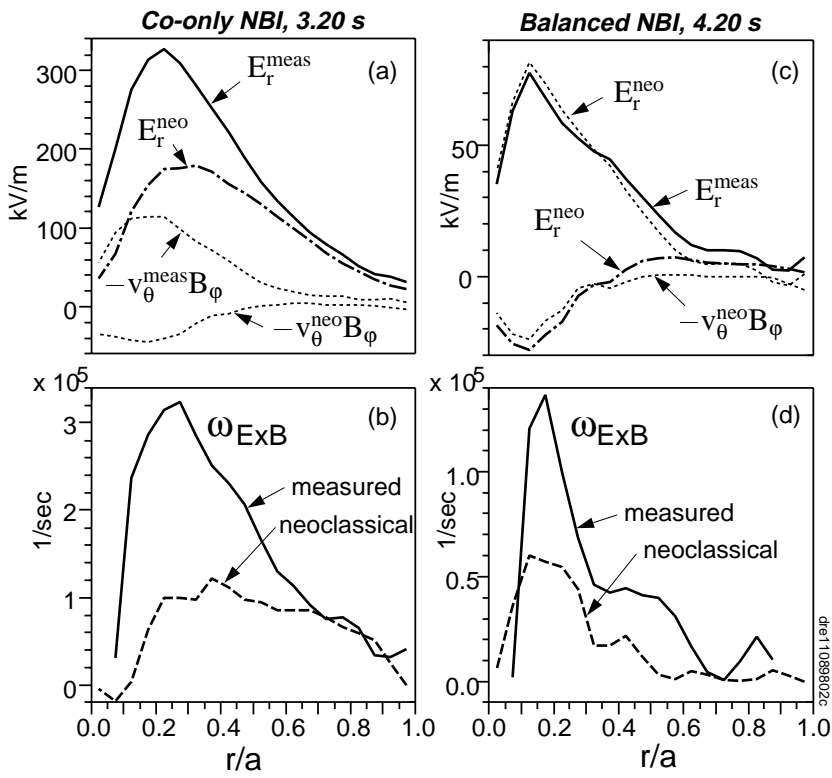

FIG. 9. Profiles before and after neutral beam torque change from co- to balanced. (a) Neoclassical and apparent measured radial electric fields for the coinjection case together with their poloidal rotation components. (b) Shearing rates corresponding to frame (a). (c) Neoclassical and apparent measured radial electric fields for the balanced injection case together with their poloidal rotation components. (d) Shearing rates corresponding to frame (c). 


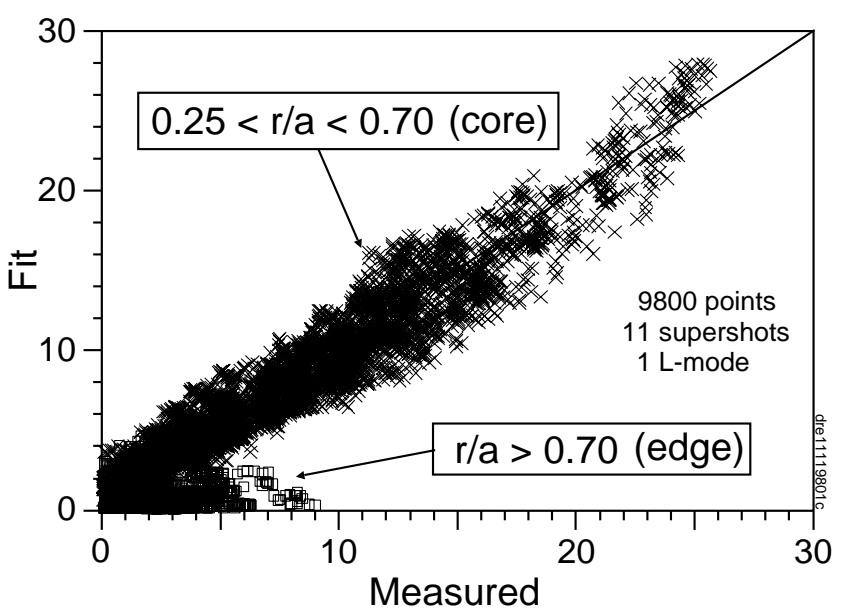

FIG. 10. Comparison of fit to apparent measured carbon poloidal rotation with apparent measured carbon poloidal rotation.

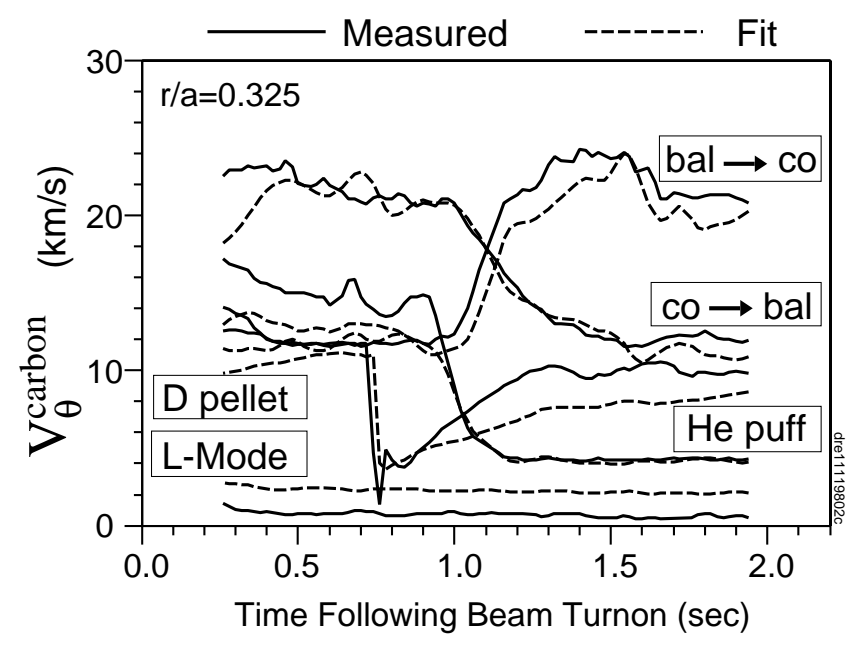

FIG. 11. Evolution of apparent measured carbon poloidal rotation closely follows $T_{i}$ in supershot perturbation experiments. Shown are beam torque changes from balanced to coinjection and from coinjection to balanced, deuterium pellet and helium puff perturbations, and one L-Mode plasma for comparison. The nominal parameters of the supershot discharges are 4.8 T, 1.6 MA, $12.1-13.5 \mathrm{MW}$, major radius $251 \mathrm{~cm}$, minor radius $86 \mathrm{~cm}$, circular cross-section, and positive magnetic shear. 


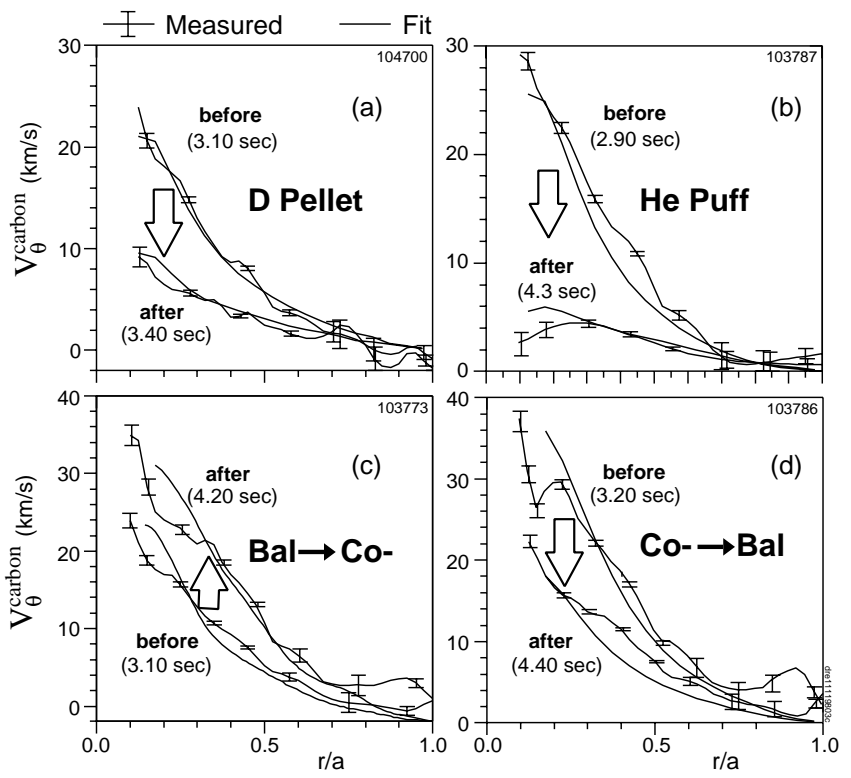

FIG. 12. Profiles of apparent measured carbon poloidal velocity closely resemble $T_{i}$ in before and after phases of the supershot perturbation experiments of Fig. 12. Neutral beam heating began at 2.5 seconds. 